Chinese Journal of Organic Chemistry

\title{
云南荵木茎中的抗炎吲哚生物碱
}

\author{
解天珍 ${ }^{\dagger}, a, b$ 赵云丽 ${ }^{\dagger}, b$ 马伟光 ${ }^{c}$ 王易芬 ${ }^{b}$ 于浩飞 ${ }^{a}$ 王 蓓 ${ }^{b}$ \\ 魏 金金 $b$ 黄之镨 $c$ 朱培凤 $b$ 刘亚平*, $b$ 罗晓东*, $a, b$ \\ $\left({ }^{a}\right.$ 云南大学化学科学与工程学院药学院 教育部自然资源药物化学重点实验室 昆明 650091) \\ $\left({ }^{b}\right.$ 中国科学院昆明植物研究所 植物化学与西部植物资源持续利用国家重点实验室 昆明 650201) \\ ( ${ }^{c}$ 云南中医药大学 昆明 650500)
}

\begin{abstract}
摘要 应用多种色谱和波谱学方法, 从云南荵木(Kopsia officinalis)茎中分离鉴定了 27 个单萜吲哚生物碱, 包括 7 个新 化合物 kopsiofficines $A \sim G$ 和 20 个已知化合物. 此外, 建立脂多糖(LPS)诱导的小鼠巨噬细胞 RAW 264.7 炎症模型, 通 过测定 IL-1 $\beta$, PGE2 和 TNF- $\alpha$ 炎症因子释放评价生物碱的抗炎活性. 结果表明, kopsiofficines A (1), kopsiofficines B (2), kopsiofficines D (4), kopsiofficines F (6), kopsiofficines G (7), 12-methoxykopsine (11), kopsinoline (15), (一)- $N$-methoxycarbonyl-11,12-methylenedioxykopsinaline (16), kopsinine (18)和 kopsinic acid (20)表现出显著的抗炎活性, 与阳性对照 (地塞米松)基本相当. 研究发现 C-5 位丙酮基取代的单萜吲哚生物碱的抗炎活性明显强于原型生物碱, 推测丙酮基可能 是抗炎活性的药效促进基团, 研究结果为进一步的结构修饰和药理学研究提供了线索.
\end{abstract}

关键词 云南荵木; kopsiofficines $\mathrm{A} \sim \mathrm{G}$; 抗炎; 单萜吲哚生物碱

\section{Anti-Inflammatory Indole Alkaloids from the Stems of Kopsia officinalis}

\author{
Xie, Tian-Zhen ${ }^{\dagger, a, b} \quad$ Zhao, Yun-Li ${ }^{\dagger}{ }^{\dagger, b} \quad$ Ma, Wei-Guang ${ }^{c} \quad$ Wang, Yi-Fen ${ }^{b} \quad$ Yu, Hao-Fei ${ }^{a}$ \\ Wang, $\mathrm{Bei}^{b} \quad$ Wei, $\mathrm{Xin}^{b} \quad$ Huang, Zhi-Pu ${ }^{c} \quad \mathrm{Zhu}$, Pei-Feng ${ }^{b}$ \\ Liu, Ya-Ping *,b Luo, Xiao-Dong*,a,b \\ ( ${ }^{a}$ Key Laboratory of Medicinal Chemistry for Natural Resource, Ministry of Education and Yunnan Province, \\ School of Chemical Science and Technology, Yunnan University, Kunming 650091) \\ $\left({ }^{b}\right.$ State Key Laboratory of Phytochemistry and Plant Resources in West China, Kunming Institute of Botany, \\ Chinese Academy of Sciences, Kunming 650201) \\ ( ${ }^{c}$ Yunnan University of Traditional Chinese Medicine, Kunming 650500)
}

\begin{abstract}
Seven new monoterpenoid indole alkaloids, kopsiofficines $A \sim G$, together with twenty known alkaloids, were isolated from the stems of Kopsia officinalis. Their structures were elucidated on the basis of extensive spectroscopic methods. The anti-inflammatory activities of all alkaloids were evaluated on lipopolysaccharide (LPS)-stimulated RAW 264.7 cells by the inhibiting the production of IL-1 $\beta$, PGE2 and TNF- $\alpha$. Among them, kopsiofficines A (1), kopsiofficines B (2), kopsiofficines D (4), kopsiofficines F (6), kopsiofficines G (7), 12-methoxykopsine (11), kopsinoline (15), (-)- $N$-methoxycarbonyl11,12-methylenedioxykopsinaline (16), kopsinine (18) and kopsinic acid (20) exhibited significant anti-inflammatory activity, which were comparable to that of dexamethasone. The results supposed that the acetonyl group at $\mathrm{C}-5$ of monoterpenoid indole alkaloids play an important role in their anti-inflammatory activity.
\end{abstract}

Keywords Kopsia officinalis; kopsiofficines A $\sim \mathrm{G}$; anti-inflammatory; monoterpenoid indole alkaloids

\footnotetext{
* Corresponding authors. E-mail: xdluo@mail.kib.ac.cn; liuyaping@mail.kib.ac.cn

Received September 26, 2019; revised November 2, 2019; November 21, 2019.

Project supported by the Yunnan Major Science and Technology Project (No. 2019ZF003), the National Key Research and Development Program of China (No. 2017YFC1704007) and the National Natural Science Foundation of China (Nos. 31872676, 31500292).

云南省重大科技计划(No. 2019ZF003)、国家重点研究发展计划(No. 2017YFC1704007)和国家自然科学基金(Nos. 31872676, 31500292)资助项目.

$\dagger$ 共同第一作者(These authors contributed equally to this work).
} 
炎症是机体对有害刺激(如病原体、感染和毒素等) 做出的防御反应 ${ }^{[1,2]}$. 同时, 大量证据表明炎症在全身性 疾病的发展中起着非常重要的作用, 如癌症、心血管疾 病、糖尿病和阿尔茨海默症等 ${ }^{[3 \sim 5]}$. 目前, 临床上治疗炎 症的药物主要是甾体激素和非甾体. 然而, 随着药物摄 入量的增加, 产生了很多的不良反应, 如骨质流失、免 疫抑制和胃肠道损伤等 ${ }^{[6,7]}$. 因此, 迫切需要研究开发安 全有效的抗炎药物.

单萜吲哚生物碱是由色氨酸和裂环马钱子苷缩合 产生的异胡香豆苷经过一系列的骨架重排衍生而来的. 因此, 该类生物碱骨架丰富, 种类繁多, 且具有广泛而 显著的生物活性 ${ }^{[8 \sim 10]}$, 多年来一直是化学家们研究的热 点. 吲哚生物碱类化合物比较重要的生物活性是抗炎, 其中一些已经被开发为抗炎药物并被广泛应用于临床, 如吲哚美辛 ${ }^{[11]}$ 、舒林酸 ${ }^{[12]}$ 和阿西美辛 ${ }^{[13]}$ 等. 云南芯木富 含单萜吲哚生物碱 ${ }^{[14]}$, 具有消炎止痛舒筋活络的功效, 民间用于治疗咽喉炎、扁桃腺炎、风湿骨痛和四肢麻 $木^{[15]}$. 为探究云南荵木中的抗炎活性成分, 我们对其茎 进行了系统的生物碱化学成分研究, 获得 7 个新的单萜 吲哚生物碱 kopsiofficines $A \sim G(\mathbf{1} \sim 7)$ 和 20 个已知的单 萜吲哚生物碱 $(\mathbf{8} \sim 27$ )(图 1). 已知生物碱通过谱图和文 献对比被鉴定为 vincadifformine $(\mathbf{8})^{[16]}$, vincadifformine $N(4)$-oxide $(\mathbf{9})^{[17]}, 11,12$-methylenedioxykopsine $(\mathbf{1 0})^{[18]}$, 12-methoxykopsine $(\mathbf{1 1})^{[19]}$, kopsifine $(\mathbf{1 2})^{[18]}$, decarbo- methoxykopsifine $(\mathbf{1 3})^{[18]}$, 5,22-dioxokopsane $(\mathbf{1 4})^{[20]}$, kopsinoline $(\mathbf{1 5})^{[21]},(-)-N$-methoxycarbonyl-11,12-methylenedioxykopsinaline $(\mathbf{1 6})^{[20]}, 11,12$-methylenedioxykopsinaline $N(4)$-oxide $(\mathbf{1 7})^{[22]}$, kopsinine $(\mathbf{1 8})^{[23]}$, 16-epikopsinine $(\mathbf{1 9})^{[24]}$, kopsinic acid $(\mathbf{2 0})^{[25]}$, pauciduridine (21) $^{[26]}, 11$-methoxykopsilongine (22) ${ }^{[20]},(-)-N$-methoxycarbonyl-12-methoxykopsinaline $\quad(\mathbf{2 3})^{[20]}, \quad$ kopsinilam $\mathbf{( 2 4}^{[27]}$, aspidofractinine $(\mathbf{2 5})^{[25]},(-)-11,12$-methylenedioxykopsinaline (26) ${ }^{[28]}$ 和 aspidofractinine-1,3-dicarboxylic acid $(27)^{[29]}$. 同时, 测定获得的 27 个单萜吲哚生物碱的 抗炎活性.

\section{1 结果与讨论}

\section{1 化合物 1 的结构鉴定}

白色无定形粉末，Dragendorff's 试剂阳性反应，推 测其可能是生物碱. 高分辨质谱 HRESIMS 给出的分子 式为 $\mathrm{C}_{26} \mathrm{H}_{28} \mathrm{~N}_{2} \mathrm{O}_{7}\left(\mathrm{~m} / z 481.1965[\mathrm{M}+\mathrm{H}]^{+}\right)$, UV 图谱中显 示结构中存在一个二氢吲哚发色团(196, 224, 244 和 290 $\mathrm{nm})$, 同时 IR 图谱中显示结构中含有 $\mathrm{NH}\left(3438 \mathrm{~cm}^{-1}\right)$ 和 羰基 $\left(1756 \mathrm{~cm}^{-1}\right)$ 基团. ${ }^{13} \mathrm{C}$ NMR 和 DEPT 谱中显示化合 物 1 含有 26 个碳共振信号(表 1), 结合 ${ }^{1} \mathrm{H}$ NMR 图谱低 场区两个氢信号 $\left[\delta_{\mathrm{H}} 6.81(\mathrm{~d}, J=7.8 \mathrm{~Hz}, 1 \mathrm{H}, \mathrm{H}-9), 6.58(\mathrm{~d}\right.$, $J=7.8 \mathrm{~Hz}, 1 \mathrm{H}, \mathrm{H}-10)]$, 可以归属为一个二取代的吲哚环 [( $\delta_{\mathrm{C}} 75.1(\mathrm{~s}, \mathrm{C}-2), 58.3(\mathrm{~s}, \mathrm{C}-7), 131.8$ (s, C-8), 115.1 (d,



1: $\mathrm{R}^{1}=\mathrm{COOCH}_{3}, \mathrm{R}^{2}=\alpha-\mathrm{CH}_{2} \mathrm{C}_{2}^{2} \mathrm{COC}^{3} \mathrm{H}_{3}$ 2: $\mathrm{R}^{1}=\mathrm{H}, \mathrm{R}^{2}=\alpha-\mathrm{CH}_{2} \mathrm{C}^{2} \mathrm{CCH}_{3}$ 10: $R^{1}=\mathrm{COOCH}_{3}, R^{2}=\mathrm{H}_{2}$ 12: $R^{1}=\mathrm{COOCH}_{3}, R^{2}=\mathrm{O}$ 13: $R^{1}=H, R^{2}=O$

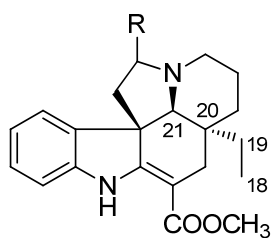

7: $\mathrm{R}=\beta-\mathrm{CH}_{2} \mathrm{COCH}_{3}$ 8: $\mathrm{R}=\mathrm{H}_{2}$ 9: $\mathrm{R}=\mathrm{H}_{2}, \mathrm{~N}(4)$-oxide

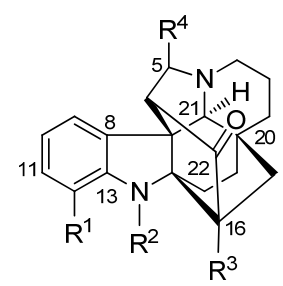

3: $\mathrm{R}^{1}=\mathrm{H}, \mathrm{R}^{2}=\mathrm{H}, \mathrm{R}^{3}=\beta-\mathrm{H}, \mathrm{R}^{4}=\alpha-\mathrm{CH}_{2} \mathrm{COCH}_{3}$ 4: $\mathrm{R}^{1}=\mathrm{OCH}_{3}, \mathrm{R}^{2}=\mathrm{COOCH}_{3}, \mathrm{R}^{3}=\alpha-\mathrm{OH}, \mathrm{R}^{4}=\alpha-$ $\mathrm{CH}_{2} \mathrm{COCH}_{3}$

11: $\mathrm{R}^{1}=\mathrm{OCH}_{3}, \mathrm{R}^{2}=\mathrm{COOCH}_{3}, \mathrm{R}^{3}=\alpha-\mathrm{OH}, \mathrm{R}^{4}=\mathrm{H}_{2}$

14: $\mathrm{R}^{1}=\mathrm{H}, \mathrm{R}^{2}=\mathrm{H}, \mathrm{R}^{3}=\alpha-\mathrm{H}, \mathrm{R}^{4}=\mathrm{O}$
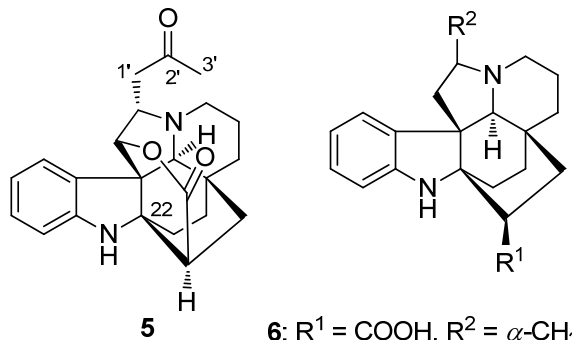

5

18: $\mathrm{R}^{1}=\mathrm{COOCH}_{3}, \mathrm{R}^{2}=\mathrm{H}_{2}$

20: $\mathrm{R}^{1}=\mathrm{COOH}, \mathrm{R}^{2}=\mathrm{H}_{2}$

24: $R^{1}=\mathrm{COOCH}_{3}, R^{2}=\mathrm{O}$

25: $\mathrm{R}^{1}=\mathrm{H}, \mathrm{R}^{2}=\mathrm{H}_{2}$

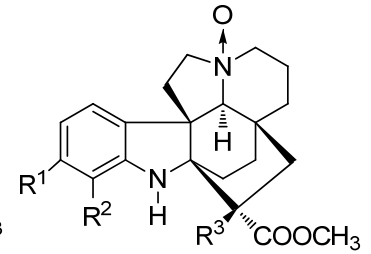

15: $R^{1}=H, R^{2}=H, R^{3}=H$ 17: $\mathrm{R}^{1}, \mathrm{R}^{2}=\mathrm{OCH}_{2} \mathrm{O}, \mathrm{R}^{3}=\mathrm{OH}$
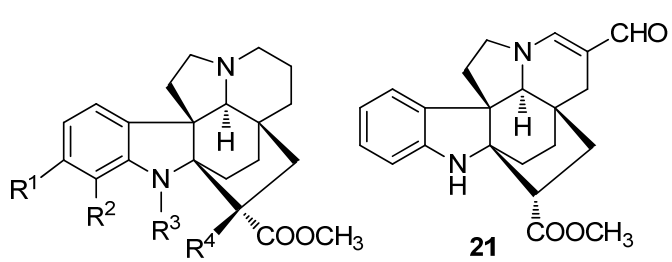

16: $R^{1}, R^{2}=\mathrm{OCH}_{2} \mathrm{O}, \mathrm{R}^{3}=\mathrm{COOCH}_{3}, \mathrm{R}^{4}=\mathrm{OH}$

19: $R^{1}, R^{2}, R^{3}, R^{4}=H$

22: $R^{1}, R^{2}=\mathrm{OCH}_{3}, R^{3}=\mathrm{COOCH}_{3}, R^{4}=\mathrm{OH}$

23: $R^{1}=H, R^{2}=\mathrm{OCH}_{3}, R^{3}=\mathrm{COOCH}_{3}, R^{4}=\mathrm{OH}$

26: $\mathrm{R}^{1}, \mathrm{R}^{2}=\mathrm{OCH}_{2} \mathrm{O}, \mathrm{R}^{3}=\mathrm{H}, \mathrm{R}^{4}=\mathrm{OH}$

图 1 化合物 $1 \sim 27$ 的结构

Figure 1 Chemical structures of compounds $\mathbf{1} \sim \mathbf{2 7}$ 
表 $\mathbf{1}$ 化合物 $\mathbf{1} \sim \mathbf{4}$ 的 ${ }^{1} \mathrm{H} \mathrm{NMR}$ 和 ${ }^{13} \mathrm{C} \mathrm{NMR}$ 波谱数据 $(J \text { 用 } \mathrm{Hz} \text { 表示 })^{a}$

Table $1{ }^{1} \mathrm{H}$ NMR and ${ }^{13} \mathrm{C}$ NMR spectroscopic data for compounds $\mathbf{1} \sim \mathbf{4}(\mathrm{J}$ in $\mathrm{Hz})$

\begin{tabular}{|c|c|c|c|c|c|c|c|c|}
\hline \multirow{2}{*}{ No. } & \multicolumn{2}{|l|}{1} & \multicolumn{2}{|l|}{2} & \multicolumn{2}{|l|}{3} & \multicolumn{2}{|l|}{4} \\
\hline & $\delta_{\mathrm{H}}$ & $\delta_{\mathrm{C}}$ & $\delta_{\mathrm{H}}$ & $\delta_{\mathrm{C}}$ & $\delta_{\mathrm{H}}$ & $\delta_{\mathrm{C}}$ & $\delta_{\mathrm{H}}$ & $\delta_{\mathrm{C}}$ \\
\hline 2 & & 75.1 & & 71.2 & & 69.2 & & 76.3 \\
\hline $3 a$ & $2.93 \sim 2.82(\mathrm{~m})$ & 45.0 & $3.65 \sim 3.55(\mathrm{~m})$ & 44.5 & $2.94 \sim 2.84(\mathrm{~m})$ & 45.2 & $2.92 \sim 2.81(\mathrm{~m})$ & 45.0 \\
\hline $3 b$ & $3.02 \sim 2.95(\mathrm{~m})$ & & $3.78(\mathrm{~d}, 13.6)$ & & $3.02 \sim 2.95(\mathrm{~m})$ & & $\begin{array}{l}2.96(\mathrm{dd}, 14.4, \\
3.8)\end{array}$ & \\
\hline 5 & $\begin{array}{l}3.69(\mathrm{dt}, 7.1 \\
4.5)\end{array}$ & 61.2 & $4.30(\mathrm{dt}, 8.0,4.0)$ & 60.1 & $3.70(\mathrm{dt}, 7.5,4.6)$ & 61.6 & $3.69(\mathrm{dt}, 7.5,4.3)$ & 61.3 \\
\hline 6 & $2.27(\mathrm{~d}, 4.5)$ & 59.5 & $2.64(\mathrm{~d}, 4.0)$ & 59.8 & $2.22(\mathrm{dd}, 4.6,1.5)$ & 63.8 & $2.35(\mathrm{~d}, 4.3)$ & 58.5 \\
\hline 7 & & 58.3 & & 58.0 & & 61.5 & & 59.5 \\
\hline 8 & & 131.8 & & 131.3 & & 133.9 & & 139.8 \\
\hline 9 & $6.81(\mathrm{~d}, 7.8)$ & 115.1 & $7.40(\mathrm{~d}, 7.7)$ & 114.6 & $7.23(\mathrm{~d}, 7.7)$ & 122.6 & $6.93(\mathrm{~d}, 8.3)$ & 114.6 \\
\hline 10 & $6.58(\mathrm{~d}, 7.8)$ & 105.2 & $7.06(\mathrm{~d}, 7.7)$ & 98.9 & $6.76(t, 7.7)$ & 119.7 & $7.10(\mathrm{t}, 8.3)$ & 127.1 \\
\hline 11 & & 149.0 & & 147.5 & $7.03(t, 7.7)$ & 127.7 & $6.80(\mathrm{~d}, 8.3)$ & 112.2 \\
\hline 12 & & 135.8 & & 131.2 & $6.63(\mathrm{~d}, 7.7)$ & 110.9 & & 150.4 \\
\hline 13 & & 123.8 & & 133.3 & & 150.8 & & 130.7 \\
\hline $14 \mathrm{a}$ & $1.26 \sim 1.20(\mathrm{~m})$ & 15.9 & $1.93(\mathrm{~d}, 13.7)$ & 15.5 & $1.26 \sim 1.18(\mathrm{~m})$ & 16.1 & $1.24 \sim 1.20(\mathrm{~m})$ & 16.1 \\
\hline $14 \mathrm{~b}$ & $1.78 \sim 1.67(\mathrm{~m})$ & & $2.59 \sim 2.48(\mathrm{~m})$ & & $1.73^{b}$ & & $1.77 \sim 1.68(\mathrm{~m})$ & \\
\hline $15 \mathrm{a}$ & $1.31^{b}$ & 33.4 & $2.10^{b}$ & 33.5 & $1.29^{b}$ & 34.1 & $1.27^{b}$ & 33.3 \\
\hline $15 b$ & $1.54^{b}$ & & $2.27 \sim 2.19(\mathrm{~m})$ & & $1.49(\mathrm{~d}, 13.6)$ & & $1.52 \sim 1.48(\mathrm{~m})$ & \\
\hline 16 & & 82.0 & & 81.1 & $2.65^{b}$ & 52.1 & & 81.9 \\
\hline $17 \mathrm{a}$ & $1.53^{b}$ & 43.3 & $2.06^{b}$ & 41.8 & $\begin{array}{l}1.59(\mathrm{ddd}, 15.1 \\
10.8,1.6)\end{array}$ & 33.0 & $\begin{array}{l}2.31(\mathrm{dd}, 15.1 \\
3.8)\end{array}$ & 42.8 \\
\hline $17 b$ & $\begin{array}{l}2.33(\mathrm{dd}, 15.1 \\
3.8)\end{array}$ & & $2.92 \sim 2.88(\mathrm{~m})$ & & $\begin{array}{l}1.95(\mathrm{dd}, 15.1, \\
1.6)\end{array}$ & & $1.55 \sim 1.50(\mathrm{~m})$ & \\
\hline $18 \mathrm{a}$ & $\begin{array}{l}1.61(\mathrm{ddd}, 13.8 \\
12.3,5.0)\end{array}$ & 19.3 & $\begin{array}{l}2.18(\mathrm{dd}, 13.4 \\
5.0)\end{array}$ & 20.1 & $1.71^{b}$ & 23.9 & $1.62 \sim 1.54(\mathrm{~m})$ & 20.1 \\
\hline $18 \mathrm{~b}$ & $2.45^{b}$ & & $2.72 \sim 2.66(\mathrm{~m})$ & & $1.78^{b}$ & & $2.43 \sim 2.33(\mathrm{~m})$ & \\
\hline $19 a$ & $1.29^{b}$ & 34.5 & $2.03^{b}$ & 35.2 & $1.30^{b}$ & 36.0 & $1.19^{b}$ & 34.5 \\
\hline $19 b$ & $1.50^{b}$ & & $2.11^{b}$ & & & & $1.48 \sim 1.42(\mathrm{~m})$ & \\
\hline 20 & & 32.2 & & 32.9 & & 31.1 & & 31.8 \\
\hline 21 & $3.25(\mathrm{~d}, 2.0)$ & 70.5 & $4.02(\mathrm{~s})$ & 69.9 & $3.42(\mathrm{~d}, 1.3)$ & 71.3 & $3.19(\mathrm{~d}, 2.0)$ & 70.1 \\
\hline 22 & & 211.9 & & 213.7 & & 216.0 & & 211.8 \\
\hline l'a & $2.47^{b}$ & 52.2 & $3.04 \sim 2.96(\mathrm{~m})$ & 55.2 & $2.67^{b}$ & 52.7 & $\begin{array}{l}2.49(\mathrm{dd}, 15.0 \\
4.7)\end{array}$ & 52.2 \\
\hline $1^{\prime} b$ & $\begin{array}{l}2.66(\mathrm{dd}, 14.9 \\
4.5)\end{array}$ & & $\begin{array}{l}3.50(\mathrm{dd}, 14.0 \text {, } \\
4.1)\end{array}$ & & $\begin{array}{l}2.47(\mathrm{dd}, 15.0 \\
7.2)\end{array}$ & & $\begin{array}{l}2.68(\mathrm{dd}, 15.0 \\
4.7)\end{array}$ & \\
\hline $2^{\prime}$ & & 207.2 & & 207.5 & & 207.5 & & 207.1 \\
\hline $3^{\prime}$ & $2.14(\mathrm{~s})$ & 31.0 & $2.88(\mathrm{~s})$ & 30.6 & $2.14(\mathrm{~s})$ & 31.0 & $2.14(\mathrm{~s})$ & 31.0 \\
\hline $\mathrm{OCH}_{2} \mathrm{O}$ & $\begin{array}{l}5.90(\mathrm{~d}, 1.4) \\
5.87(\mathrm{~d}, 1.4)\end{array}$ & 100.6 & $\begin{array}{l}6.69(\mathrm{~d}, 0.7) \\
6.61(\mathrm{~d}, 0.7)\end{array}$ & 100.3 & & & & \\
\hline $\mathrm{NCOOCH}_{3}$ & $3.76(\mathrm{~s})$ & 53.6 & & & & & $3.66(\mathrm{~s})$ & 53.5 \\
\hline $\mathrm{NCOOCH}_{3}$ & & 155.5 & & & & & & 156.2 \\
\hline $\mathrm{OCH}_{3}$ & & & & & & & $3.79(\mathrm{~s})$ & 55.9 \\
\hline $\mathrm{OH}$ & $7.01(\mathrm{~s})$ & & $6.30(\mathrm{~s})$ & & & & $6.91(\mathrm{~s})$ & \\
\hline $\mathrm{NH}$ & & & $6.10(\mathrm{~s})$ & & $3.55(\mathrm{~s})$ & & & \\
\hline
\end{tabular}

${ }^{a}$ Compounds $\mathbf{1 , 3}$ and $\mathbf{4}$ were measured in $\mathrm{CDCl}_{3}$, and $\mathbf{2}$ was measured in DMSO- $d_{6} \cdot{ }^{b}$ Overlapped.

C-9), 105.2 (d, C-10), 149.0 (s, C-11), 135.8 (s, C-12), 123.8 (s, C-13)]. 同时, 结构中还含有两个酮羰基 $\left(\delta_{\mathrm{C}}\right.$ $211.9,207.2)$ 、一个酰胺羰基 $\left(\delta_{\mathrm{C}} 155.5\right)$ 、一个亚甲二氧 基 $\left(\delta_{\mathrm{C}} 100.6\right)$ 、一个甲氧基 $\left[\delta_{\mathrm{C}} 53.6 / \delta_{\mathrm{H}} 3.76(\mathrm{~s})\right]$ 和一个甲基
$\left[\delta_{\mathrm{C}} 31.0 / \delta_{\mathrm{H}} 2.14(\mathrm{~s})\right]$. 从已经报道的该属植物中生物碱 数据来看, 化合物 $\mathbf{1}$ 是一个莣木素类型的单萜吲哚生物 碱, 且碳谱数据与已知生物碱 11,12-methylenedioxykopsine (10) ${ }^{[18]}$ 非常相似. 只是化合物 $\mathbf{1}$ 多了三个碳信号 
$\left[\delta_{\mathrm{C}} 52.2(\mathrm{t}), 207.2(\mathrm{~s})\right.$ 和 $\left.31.0(\mathrm{q})\right]$, 这也与化合物 $\mathbf{1}$ 的质谱 相吻合. 在 $\mathrm{HMBC}$ 图谱中, 质子信号 $\delta_{\mathrm{H}} 2.14(\mathrm{~s}, 3 \mathrm{H}$, H-3') 与碳信号 $\delta_{\mathrm{C}} 207.2\left(\mathrm{~s}, \mathrm{C}-2^{\prime}\right)$ 和 $52.2\left(\mathrm{t}, \mathrm{C}-1^{\prime}\right)$ 相关, 质 子信号 $\delta_{\mathrm{H}} 2.66\left(\mathrm{dd}, J=14.9,4.5 \mathrm{~Hz}, 1 \mathrm{H}, \mathrm{H}-\mathrm{1}^{\prime} \mathrm{b}\right)$ 与碳信号 $\delta_{\mathrm{C}} 207.2\left(\mathrm{~s}, \mathrm{C}-2^{\prime}\right), 61.2(\mathrm{~d}, \mathrm{C}-5)$ 和 59.5 (d, C-6)相关, 揭示 了 C-1', C-2'及 C-3'之间形成了一个 $\mathrm{CH}_{2} \mathrm{COCH}_{3}$ 结构片 段, 且与 C-5 位相连(图 2). 化合物 $\mathbf{1}$ 的相对构型通过 ROESY 图谱来解析(图 2), 首先 ROESY 相关信号 $\delta_{\mathrm{H}}$ $6.81(\mathrm{~d}, J=7.8 \mathrm{~Hz}, 1 \mathrm{H}, \mathrm{H}-9) / \delta_{\mathrm{H}} 3.25(\mathrm{~d}, J=2.0 \mathrm{~Hz}, 1 \mathrm{H}$, $\mathrm{H}-21)$ 和 $\delta_{\mathrm{H}} 3.25(\mathrm{~d}, J=2.0 \mathrm{~Hz}, 1 \mathrm{H}, \mathrm{H}-21) / \delta_{\mathrm{H}} 2.93 \sim 2.82$ $(\mathrm{m}, 1 \mathrm{H}, \mathrm{H}-3 \mathrm{a})$ 可以把 C-3 位的两个氢原子区别开, 此后 相关信号 $\delta_{\mathrm{H}} 3.02 \sim 2.95(\mathrm{~m}, 1 \mathrm{H}, \mathrm{H}-3 \mathrm{~b}) / \delta_{\mathrm{H}} 3.69(\mathrm{dt}, J=7.1$, $4.5 \mathrm{~Hz}, 1 \mathrm{H}, \mathrm{H}-5$ ) 表明 H-5 为 $\beta$ 构型, 由此推测与 C-5 相 连的 $\mathrm{CH}_{2} \mathrm{COCH}_{3}$ 基团为 $\alpha$ 构型. 另外相关信号 $\delta_{\mathrm{H}} 3.25$ (d, $J=2.0 \mathrm{~Hz}, 1 \mathrm{H}, \mathrm{H}-21$ ) $/ \delta_{\mathrm{H}} 1.61$ (ddd, $J=13.8,12.3,5.0$ $\mathrm{Hz}, 1 \mathrm{H}, \mathrm{H}-18 \mathrm{a}$ ), $\delta_{\mathrm{H}} 2.45$ (overlapped, $1 \mathrm{H}, \mathrm{H}-18 \mathrm{~b}$ ) $/ \delta_{\mathrm{H}} 7.01$ $(\mathrm{s}, 1 \mathrm{H}, \mathrm{OH}-16)$ 表明 $\mathrm{OH}-16$ 为 $\alpha$ 构型(图 2). 通过二维核 磁共振数据(HSQC, HMBC 和 ROESY)最终推测其结构, 并将其命名为 kopsiofficine A.
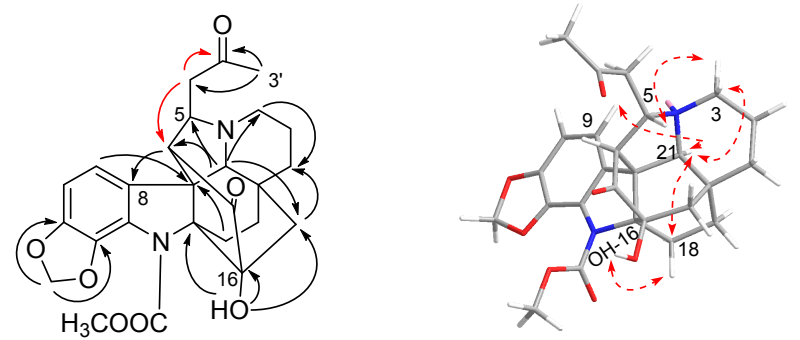

图 2 化合物 1 的主要 $\operatorname{HMBC}(\cdots)$ ) 相关和主要 ROESY $\left(r^{-\cdots}\right.$ ) 相关

Figure 2 Selective HMBC ( ... $)$ ) and ROESY (r y ) correlations of 1

\section{2 化合物 2 的结构鉴定}

根据高分辨质谱(HRESIMS)给出其准分子离子峰 $m / z: 423.1914[\mathrm{M}+\mathrm{H}]^{+}$(calcd 423.1914). 结合 ${ }^{13} \mathrm{C} \mathrm{NMR}$ 和 DEPT 谱数据(表 1) 确定分子式为 $\mathrm{C}_{24} \mathrm{H}_{26} \mathrm{~N}_{2} \mathrm{O}_{5}$. 化合物 $\mathbf{2}$ 的 1D NMR 数据与化合物 $\mathbf{1}$ 的很接近, 不同之处在于 化合物 2 比化合物 1 少了一个甲酯基信号. 同时质谱上 少了 58 个道尔顿也验证了上述推测. 在 $\mathrm{HMBC}$ 图谱中, 吲哚氮氢质子信号 $\delta_{\mathrm{H}} 6.10(\mathrm{~s}, 1 \mathrm{H})$ 与碳信号 $\delta_{\mathrm{C}} 71.2(\mathrm{~s}$, C-2), 58.0 (s, C-7), 131.3 (s, C-8) 和 133.3 (s, C-13)的相关 也验证了化合物 2 中吲哚环氮原子上连接的是一个氢原 子而不是甲酯基. ROESY 相关点 $\delta_{\mathrm{H}} 7.40(\mathrm{~d}, J=7.7 \mathrm{~Hz}$, $1 \mathrm{H}, \mathrm{H}-9) / \delta_{\mathrm{H}} 3.50\left(\mathrm{dd} J=14.0,4.1 \mathrm{~Hz}, 1 \mathrm{H}, \mathrm{H}-1^{\prime} \mathrm{b}\right) / \delta_{\mathrm{H}} 4.02$ $(\mathrm{s}, 1 \mathrm{H}, \mathrm{H}-21), \delta_{\mathrm{H}} 4.02(\mathrm{~s}, 1 \mathrm{H}, \mathrm{H}-21) / \delta_{\mathrm{H}} 2.18(\mathrm{dd}, J=13.4$, $5.0 \mathrm{~Hz}, 1 \mathrm{H}, \mathrm{H}-18 \mathrm{a}), \delta_{\mathrm{H}} 2.72 \sim 2.66(\mathrm{~m}, 1 \mathrm{H}, \mathrm{H}-18 \mathrm{~b}) / \delta_{\mathrm{H}} 6.3$ (s, 1H, OH-16), 表明它们处于同一平面, 并且化合物 1
和 2 具有相似的比旋光值 $\left([\alpha]_{D}^{22.4}+23\right.$ for $\mathbf{1},[\alpha]_{D}^{22.4}+21$ for 2), 说明化合物 $\mathbf{2}$ 的绝对构型也与化合物 $\mathbf{1}$ 相同.

\section{3 化合物 3 的结构鉴定}

高分辨质谱 HR-ESI-MS 给出化合物 $\mathbf{3}$ 的分子式为 $\mathrm{C}_{23} \mathrm{H}_{26} \mathrm{~N}_{2} \mathrm{O}_{2}\left(\mathrm{~m} / \mathrm{z} 363.2064[\mathrm{M}+\mathrm{H}]^{+}\right)$. 其 $\mathrm{UV}$ 数据 242, $295 \mathrm{~nm}$ 和 IR 数据 $3439,1632,1611,1089 \mathrm{~cm}^{-1}$ 表明化合 物 3 的结构中存在吲哚环 ${ }^{[22]} .{ }^{13} \mathrm{C}$ NMR 和 DEPT 显示化 合物 3 有 23 个碳信号, 包括一个甲基 $\left(\delta_{\mathrm{C}} 31.0\right)$ 、七个亚 甲基 $\left(\delta_{\mathrm{C}} 16.1,23.9,33.0,34.1,36.0,45.2,52.7\right)$ 、八个次甲 基 $\left(\delta_{\mathrm{C}} 52.1,61.6,63.8,71.3,110.9,119.7,122.6,127.7\right)$ 和 七个季碳 $\left(\delta_{\mathrm{C}} 31.1,61.5,69.2,133.9,150.8,207.5,216.0\right)$. 化合物 3 的碳谱数据与蕊木素类型已知生物碱 $(-)-k o p s a n o n e^{[18]}$ 非常相似, 不同之处在于化合物 3 多 三个碳信号 $\left[\delta_{\mathrm{C}} 31.0(\mathrm{q}), 207.5(\mathrm{~s})\right.$ 和 $\left.52.7(\mathrm{t})\right]$. 通过 $\mathrm{HMBC}$ 相关信号 $\delta_{\mathrm{H}} 2.14\left(\mathrm{~s}, 3 \mathrm{H}, \mathrm{H}-3^{\prime}\right) / \delta_{\mathrm{C}} 207.5\left(\mathrm{~s}, \mathrm{C}-2^{\prime}\right)$ 和 52.7 (t, C-1') 表明化合物 3 的结构中含有 $\mathrm{CH}_{3} \mathrm{COCH}_{2}$ 结 构片段, 且 $\mathrm{HMBC}$ 图谱中 $\delta_{\mathrm{H}} 2.47(\mathrm{dd}, J=15.0,7.2 \mathrm{~Hz}$, $1 \mathrm{H}, \mathrm{H}-1$ 'b)与 $\delta_{\mathrm{C}} 61.6(\mathrm{~d}, \mathrm{C}-5)$ 和 $\delta_{\mathrm{C}} 63.8(\mathrm{~d}, \mathrm{C}-6)$ 相关说明 该基团连接在 C-5 位. 化合物 $\mathbf{3}$ 的相对构型通过 ROESY 图谱解析, 由于荵木素类生物碱 H-21 的相对构型通过 生源途径可以确定为 $\alpha$ 构型, 而相关信号 $\delta_{\mathrm{H}} 3.42(\mathrm{~d}, J=$ $1.3 \mathrm{~Hz}, 1 \mathrm{H}, \mathrm{H}-21) / \delta_{\mathrm{H}} 2.94 \sim 2.84(\mathrm{~m}, 1 \mathrm{H}, \mathrm{H}-3 \mathrm{a})$ 表明 $\mathrm{H}-3 \mathrm{a}$ 为 $\alpha$ 构型, 而 C-3 上另外一个氢原子 $\mathrm{H}-3 \mathrm{~b}$ 为 $\beta$ 构型. ROESY 相关信号 $\delta_{\mathrm{H}} 3.02 \sim 2.95(\mathrm{~m}, 1 \mathrm{H}, \mathrm{H}-3 \mathrm{~b}) / \delta_{\mathrm{H}} 3.70$ $(\mathrm{dt}, J=7.5,4.6 \mathrm{~Hz}, 1 \mathrm{H}, \mathrm{H}-5), \delta_{\mathrm{H}} 3.02 \sim 2.95(\mathrm{~m}, 1 \mathrm{H}$, $\mathrm{H}-3 \mathrm{~b}$ ) $/ \delta_{\mathrm{H}} 2.65$ (overlapped, $1 \mathrm{H}, \mathrm{H}-16$ ), 表明 H-5 和 H-16 都是 $\beta$ 构型, 从而推测 $\mathrm{C}-5$ 上连接的 $\mathrm{CH}_{3} \mathrm{COCH}_{2}$ 基团为 $\alpha$ 构型. 仔细分析化合物 $\mathbf{3}$ 的二维核磁共振数据(HSQC, $\mathrm{HMBC}, 1 \mathrm{H}-1 \mathrm{H}$ COSY, ROESY) 推导了 3 的结构如图 1 所 示, 并将其命名为 kopsiofficine C.

\section{4 化合物 4 的结构鉴定}

高分辨质谱 HRESIMS 给出其分子离子峰 $[\mathrm{M}-\mathrm{H}]^{-}$ $m / z 465.2042$, 确定其分子式为 $\mathrm{C}_{26} \mathrm{H}_{30} \mathrm{~N}_{2} \mathrm{O}_{6}$, 根据化合物 4 的 ${ }^{1} \mathrm{H} \mathrm{NMR},{ }^{13} \mathrm{C}$ NMR 和 DEPT 波谱数据发现其具有一 个取代的吲哚环结构. 比较化合物 $\mathbf{4}$ 与 $\mathbf{1}$ 的 1D-NMR 数 据(表 1)发现, 它们的结构非常相似. 不同的是化合物 $\mathbf{4}$ 少了一个亚甲二氧基 $\left(\delta_{\mathrm{C}} 100.6, \mathrm{t}\right)$ 信号, 多了一个甲氧基 [ $\left.\delta_{\mathrm{C}} 55.9(\mathrm{q}) / \delta_{\mathrm{H}} 3.79(\mathrm{~s})\right]$. 通过 HMBC 图谱中 $\delta_{\mathrm{H}} 3.79(\mathrm{~s}$, $3 \mathrm{H})$ 与 $\delta_{\mathrm{C}} 150.4(\mathrm{~s}, \mathrm{C}-12)$ 相关, $\delta_{\mathrm{H}} 6.93(\mathrm{~d}, J=8.3 \mathrm{~Hz}, 1 \mathrm{H}$, $\mathrm{H}-9)$ 与 $\delta_{\mathrm{C}} 112.2(\mathrm{~d}, \mathrm{C}-11)$ 和 $130.7(\mathrm{~s}, \mathrm{C}-13), \delta_{\mathrm{H}} 6.80(1 \mathrm{H}$, $\mathrm{d}, J=8.3 \mathrm{~Hz}, \mathrm{H}-11)$ 与 $\delta_{\mathrm{C}} 130.7$ (s, C-13)相关, 证明该甲 氧基连接在 C-12 位. 详细比较分析化合物 $\mathbf{4}$ 的二维核磁 共振数据, 确定其他部分与化合物 $\mathbf{1}$ 相同. 


\section{5 化合物 5 的结构鉴定}

高分辨质谱 HRESIMS 给出的准分子离子峰 $\mathrm{m} / \mathrm{z}$ $401.1836[\mathrm{M}+\mathrm{Na}]^{+}$, 结合 ${ }^{1} \mathrm{H}$ NMR, ${ }^{13} \mathrm{C}$ NMR 确定化合 物 5 的分子式为 $\mathrm{C}_{23} \mathrm{H}_{26} \mathrm{~N}_{2} \mathrm{O}_{3}$, 计算得不饱和度为 12 . 其 ${ }^{13} \mathrm{C}$ NMR 和 DEPT 数据显示结构中有 23 个碳信号, 包 括一个甲基 $\left(\delta_{\mathrm{C}} 30.9\right)$ 、七个亚甲基 $\left(\delta_{\mathrm{C}} 23.0,29.5,32.5\right.$, $33.0,33.7,41.7,47.6)$ 、八个次甲基 $\left(\delta_{\mathrm{C}} 39.5,57.9,69.8\right.$, $90.2,112.2,120.2,122.6,128.4)$ 和七个季碳 $\left(\delta_{\mathrm{C}} 32.9,50.1\right.$, $66.0,132.9,150.2,173.5,207.1$ )(表 2), 这与荵木素类已 知生物碱 paucidactine $\mathrm{E}^{[26]}$ 的碳谱数据非常相似, 仔细 比较这两个化合物的 1D-NMR 数据发现, 5 多了 3 个碳 信号 $\left[\delta_{\mathrm{C}} 41.7\right.$ (t, C-1'), 207.1 (s, C-2') 和 30.9 (q, C-3')], 推 测化合物 5 可能是其丙酮基衍生物. 通过 $\mathrm{HMBC}$ 相关信 号 $\delta_{\mathrm{H}} 2.76\left(1 \mathrm{H}, \mathrm{dd}, J=18.2,5.9 \mathrm{~Hz}, \mathrm{H}-1^{\prime}-b\right) / \delta_{\mathrm{C}} 207.1(\mathrm{~s}$, C-2'), $90.2(\mathrm{~d}, \mathrm{C}-6)$ 和 $57.9(\mathrm{~d}, \mathrm{C}-5), \delta_{\mathrm{H}} 2.51(\mathrm{~s}, 3 \mathrm{H}$, $\left.\mathrm{H}-3^{\prime}\right) / \delta_{\mathrm{C}} 41.7$ (t, C-1'), 以及 ${ }^{1} \mathrm{H}-{ }^{1} \mathrm{H}$ COSY 图谱中 3.34 $3.23(\mathrm{~m}, 1 \mathrm{H}, \mathrm{H}-5)$ 与 $\delta_{\mathrm{H}} 4.18 \sim 4.14(\mathrm{~m}, 1 \mathrm{H}, \mathrm{H}-6)$ 和 2.76 (dd, $J=18.2,5.9 \mathrm{~Hz}, 1 \mathrm{H}, \mathrm{H}-1$ 'b) 相关, 证实了上述推测, 且该丙酮基连接在 C-5 位(图 3). 通过 ROESY 图谱, $\delta_{\mathrm{H}}$ $2.64 \sim 2.59\left(\mathrm{~m}, 1 \mathrm{H}, \mathrm{H}-1^{\prime} \mathrm{a}\right) / \delta_{\mathrm{H}} 2.54(\mathrm{~s}, 1 \mathrm{H}, \mathrm{H}-21)$ 以及 $\delta_{\mathrm{H}}$ $2.54(\mathrm{~s}, 1 \mathrm{H}, \mathrm{H}-21) / \delta_{\mathrm{H}} 1.64$ (overlapped, $\left.1 \mathrm{H}, \mathrm{H}-18 \mathrm{~b}\right) / \delta_{\mathrm{H}}$ $2.90 \sim 2.84(\mathrm{~m}, 1 \mathrm{H}, \mathrm{H}-16)$ 相关, 表明它们在同一平面, 且为 $\alpha$ 构型. 因此, C-5 位连接的丙酮基为 $\alpha$ 构型, 进而 推测 C-5 位连接的氢原子 H-5 为 $\beta$ 构型. 由此, 推导了 kopsiofficine E (5)的结构如图 1 所示.


图 3 化合物 $\mathbf{5}$ 的主要 $\mathrm{HMBC}(\cdots)$ 相关, 主要 ${ }^{1} \mathrm{H}-{ }^{1} \mathrm{H} \operatorname{COSY}$ (一)和 ROESY (

Figure 3 Selective $\operatorname{HMBC}(\cdots), 1 \mathrm{H}-{ }^{1} \mathrm{H} \operatorname{COSY}($ - $)$ and ROESY (

\section{6 化合物 6 的结构鉴定}

白色无定形粉末, 高分辨质谱 HRESIMS ([M+ H] ${ }^{+}, m / z$ 381.2174)推导出其分子式为 $\mathrm{C}_{23} \mathrm{H}_{28} \mathrm{~N}_{2} \mathrm{O}_{3}$, 其 $\mathrm{UV}$ 数据 203, 225, $285 \mathrm{~nm}$ 处的吸收表明, 化合物 $\mathbf{6}$ 是一个二 氢吲哚类生物碱 ${ }^{[30]}$. 通过比较化合物 $\mathbf{6}$ 与白坚木碱型已 知生物碱 kopsinic acid $(\mathbf{2 0})^{[25]}$ 的 ${ }^{1} \mathrm{H}$ NMR 和 ${ }^{13} \mathrm{C}$ NMR 数 据(表 2)发现, 化合物 $\mathbf{6}$ 比 20 多了 3 个碳信号 $\left[\delta_{\mathrm{C}} 51.7\right.$ (t, C-1'), 207.7 (s, C-2') 和 30.7 (q, C-3')], 推测结构中多了一 个丙酮基. HMBC 相关信号 $\delta_{\mathrm{H}} 2.09\left(\mathrm{~s}, 3 \mathrm{H}, \mathrm{H}-3^{\prime}\right) / \delta_{\mathrm{C}} 207.7$ (s, C-2') 和 $51.7\left(\mathrm{t}, \mathrm{C}-1^{\prime}\right), \delta_{\mathrm{H}} 2.71(\mathrm{dd}, J=6.0,6.5 \mathrm{~Hz}, 1 \mathrm{H}$, $\left.\left.\mathrm{H}-1 \mathrm{~b}^{\prime}\right)\right) / \delta_{\mathrm{C}} 56.8(\mathrm{~d}, \mathrm{C}-5)$, 证实了此丙酮基的存在, 并且 连接在 C-5 位. 从生源途径可以确定白坚木碱型生物碱 $\mathrm{H}-21$ 位的相对构型为 $\alpha$ 构型. 在 ROESY 相关信号 $\delta_{\mathrm{H}}$ $3.24(\mathrm{~s}, 1 \mathrm{H}, \mathrm{H}-21) / \delta_{\mathrm{H}} 2.89 \sim 2.84(\mathrm{~m}, 1 \mathrm{H}, \mathrm{H}-3 \mathrm{a}), \delta_{\mathrm{H}} 3.24$ $(\mathrm{s}, 1 \mathrm{H}, \mathrm{H}-21) / \delta_{\mathrm{H}} 1.97 \sim 1.90(\mathrm{~m}, 1 \mathrm{H}, \mathrm{H}-18 \mathrm{~b}), \delta_{\mathrm{H}} 1.36$ (overlapped, $1 \mathrm{H}, \mathrm{H}-18 \mathrm{a}) / \delta_{\mathrm{H}} 2.81$ (overlapped, $1 \mathrm{H}, \mathrm{H}-16$ )表 明, 它们在同一平面. 因此, H-3a 和 H-16 均为 $\alpha$ 构型, 由此推测 C-3 位链接的另一个氢原子 $\mathrm{H}-3 \mathrm{~b}$ 和 C-16 链接 的 $\mathrm{COOH}$ 基团均为 $\beta$ 构型, ROESY 相关信号 $\delta_{\mathrm{H}} 3.0(\mathrm{~d}$, $J=14.4 \mathrm{~Hz}, 1 \mathrm{H}, \mathrm{H}-3 \mathrm{~b}) / \delta_{\mathrm{H}} 3.87 \sim 3.74(\mathrm{~m}, 1 \mathrm{H}, \mathrm{H}-5)$ 表明 $\mathrm{H}-5$ 为 $\beta$ 构型，由此推测 C-5 位连接的丙酮基为 $\alpha$ 构型. 因此, 推导了 kopsiofficine F (6) 的结构, 如图 1 所示.

\section{7 化合物 7 的结构鉴定}

高分辨质谱 HRESIMS 给出化合物 7 的分子式为 $\mathrm{C}_{24} \mathrm{H}_{30} \mathrm{~N}_{2} \mathrm{O}_{3}\left(m / z 395.2326[\mathrm{M}+\mathrm{H}]^{+}\right)$. UV 数据给出化合 物的最大吸收值为 205,299 和 $326 \mathrm{~nm}$, 结合 ${ }^{13} \mathrm{C} N M R$ 数据 $\delta_{\mathrm{C}} 167.7(\mathrm{~s}, \mathrm{C}-2)$ 和 $92.4(\mathrm{~s}, \mathrm{C}-16)$, 表明化合物有 $\beta$ 苯胺丙烯酸酯发色团 ${ }^{[31]}$. 其 IR 图谱显示 $\mathrm{NH}(3426 \mathrm{~cm}$ ${ }^{1}$ )和共轭酯基 $\left(1667 \mathrm{~cm}^{-1}\right)$. 同时 ${ }^{13} \mathrm{C} \mathrm{NMR}$ 数据给出了丙 烯酯双键上碳的特征信号 $\delta_{\mathrm{C}} 167.7$ (s, C-2)和 92.4 (s, C-16). ${ }^{1} \mathrm{H}$ NMR 图谱数据(表 2)显示化合物 7 中含有邻二 取代的苯环 $\left[\delta_{\mathrm{H}} 7.18(\mathrm{~d}, J=7.4 \mathrm{~Hz}, 1 \mathrm{H}, \mathrm{H}-9), 6.84\right.$ (t, $J=$ $7.4 \mathrm{~Hz}, 1 \mathrm{H}, \mathrm{H}-10), 7.09$ (t, $J=7.4 \mathrm{~Hz}, 1 \mathrm{H}, \mathrm{H}-11), 6.75$ (d, $J=7.4 \mathrm{~Hz}, 1 \mathrm{H}, \mathrm{H}-12)]$ 、一个甲氧基 $\left[\delta_{\mathrm{H}} 3.73(\mathrm{~s}, 3 \mathrm{H})\right]$ 和两 个甲基 $\left[\delta_{\mathrm{H}} 2.10\left(\mathrm{~s}, 3 \mathrm{H}, \mathrm{H}-3^{\prime}\right), 0.55\right.$ (overlapped, $3 \mathrm{H}$, $\mathrm{H}-18)] .{ }^{13} \mathrm{C}$ NMR 和 DEPT 图谱显示, 化合物 7 含有 24 个碳信号，其中三个甲基、七个亚甲基、六个次甲基和 八个季碳(表 2). 1D-NMR 数据显示化合物 7 属于白坚木 碱型的吲哚生物碱, 且与已知化合物 vincadifformine $(8)^{[16]}$ 非常相似，只是化合物 7 在 C-5 位多一个丙酮基 $\left[\delta_{\mathrm{C}}\right.$ 31.0 (q, C-3'), $207.3\left(\mathrm{~s}, \mathrm{C}-2^{\prime}\right)$ 和 46.7 (t, C-1')]. 通过 $\mathrm{HMBC}$ 图谱, 相关信号 $\delta_{\mathrm{H}} 2.10\left(\mathrm{~s}, 3 \mathrm{H}, \mathrm{H}-3^{\prime}\right)$ 与 $\delta_{\mathrm{C}} 207.3$ (s, C-2') 和 $46.7\left(\mathrm{t}, \mathrm{C}-1^{\prime}\right)$, 相关信号 $\delta_{\mathrm{H}} 2.32(\mathrm{dd}, J=16.4$, $5.7 \mathrm{~Hz}, 1 \mathrm{H}, \mathrm{H}-1$ 'a) $/ \delta_{\mathrm{C}} 56.0(\mathrm{~d}, \mathrm{C}-5)$ 和 51.4 (t, C-6)(图 4)也 证实结构中含有一个丙酮基, 且连接在 C-5 位. ROESY 谱相关信号 $\delta_{\mathrm{H}} 2.58(\mathrm{~s}, 1 \mathrm{H}, \mathrm{H}-21) / \delta_{\mathrm{H}} 3.05(\mathrm{td}, J=10.9,5.7$ $\mathrm{Hz}, 1 \mathrm{H}, \mathrm{H}-5)$ 推测 $\mathrm{H}-5$ 为 $\alpha$ 构型, 进一步推测 $\mathrm{C}-5$ 上相连 的丙酮基团为 $\beta$ 构型. ROESY 图谱上的相关点 $\delta_{\mathrm{H}} 2.58$ $(\mathrm{s}, 1 \mathrm{H}, \mathrm{H}-21) / \delta_{\mathrm{H}} 0.57$ (overlapped, 1H, H-19a) 说明化合物 $\mathbf{7}$ 的相对构型与 8 一致(图 4). 由此推导了化合物 7 的结 构, 如图 1 所示, 并命名为 kopsiofficine G.

\section{8 生物碱 1 27 的抗炎活性}

通过脂多糖诱导的 RAW 264.7 细胞, 测定其中 IL- 

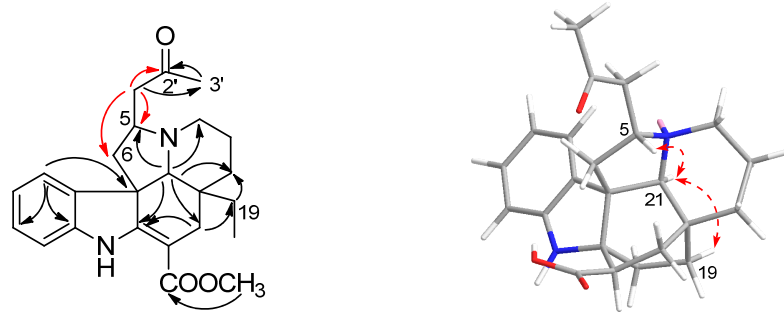

图 4 化合物 7 的主要 $\mathrm{HMBC}(\cdots)$ 相关和主要 ROESY (

Figure 4 Selective HMBC (...), and ROESY ( lations of 7

$1 \beta$, PGE2 和 TNF- $\alpha$ 炎症因子的释放, 进而评价所有生物 碱 1 27 的抗炎活性, 结果如表 3 所示. 在仅有阳性对 照(地塞米松)(上海现代哈森(商丘)药业有限公司, 批号:
1703170231)一半浓度 $(5 \mu \mathrm{g} / \mathrm{mL})$ 的条件下，生物碱 $\mathbf{1 ,}, \mathbf{2}$, $4,6,7,11,15,16,18$ 和 20 的抗炎活性略高于阳性对照 (DXM，10 $\mu \mathrm{g} / \mathrm{mL}$ )(表 3). 同时, 与脂多糖诱导组相比, 生物碱 1,4 和 7 对 IL-1 $\beta$ 炎症因子的释放表现出更强的 抑制作用 $(p<0.05 / 0.01)$; 生物碱 1, 2, 6 和 7 显著抑制 PGE2 炎症因子的释放 $(p<0.05 / 0.01)$; 生物碱 11 和 20 显著抑制 TNF- $\alpha$ 炎症因子的释放 $(p<0.05 / 0.01)$. 通过结 构分析, 发现化合物 1, 4, 6, 7 分别比化合物 10, 11, 20,8 在 C-5 位多了一个丙酮基，但是化合物 1, 4, 6, 7 的抗炎 活性明显高于化合物 $10,11,20,8$. 因此，推测丙酮基可 能是抗炎活性的药效促进基团。此外，通过噻唑蓝 (MTT)法评估了这些化合物对 RAW 264.7 的细胞毒性, 结果显示它们在相同浓度 $(5 \mu \mathrm{g} / \mathrm{mL})$ 下没有抑制 RAW

表 2 化合物 5 7 的 ${ }^{1} \mathrm{H} \mathrm{NMR}$ 和 ${ }^{13} \mathrm{C} \mathrm{NMR}$ 波谱数据 ${ }^{a}(J$ 用 $\mathrm{Hz}$ 表示 $)$

Table $2{ }^{1} \mathrm{H}$ NMR and ${ }^{13} \mathrm{C}$ NMR spectroscopic data of compounds $5 \sim 7(J$ in $\mathrm{Hz})$

\begin{tabular}{|c|c|c|c|c|c|c|}
\hline \multirow{2}{*}{ No } & \multicolumn{2}{|l|}{5} & \multicolumn{2}{|l|}{6} & \multicolumn{2}{|l|}{7} \\
\hline & $\delta_{\mathrm{H}}$ & $\delta_{\mathrm{C}}$ & $\delta_{\mathrm{H}}$ & $\delta_{\mathrm{C}}$ & $\delta_{\mathrm{H}}$ & $\delta_{\mathrm{C}}$ \\
\hline 2 & & 66.0 & & 66.0 & & 167.7 \\
\hline $3 a$ & $2.44^{b}$ & 47.6 & $2.89 \sim 2.84(\mathrm{~m})$ & 45.5 & $2.25 \sim 2.18(\mathrm{~m})$ & 48.2 \\
\hline $3 b$ & $2.81(\mathrm{dd}, 10.9,5.2)$ & & $3.0(\mathrm{~d}, 14.4)$ & & $2.94 \sim 2.86(\mathrm{~m})$ & \\
\hline 5 & $3.34 \sim 3.23(\mathrm{~m})$ & 57.9 & $3.87 \sim 3.74(\mathrm{~m})$ & 56.8 & $3.05(\mathrm{td}, 10.9,5.7)$ & 56.0 \\
\hline $6 a$ & $4.18 \sim 4.14(\mathrm{~m})$ & 90.2 & $1.41^{b}$ & 42.9 & $1.62 \sim 1.57(\mathrm{~m})$ & 51.4 \\
\hline $6 \mathrm{~b}$ & & & $2.78^{b}$ & & $1.85(\mathrm{dd}, 11.4,5.7)$ & \\
\hline 7 & & 50.1 & & 56.3 & & 53.6 \\
\hline 8 & & 132.9 & & 140.3 & & 137.5 \\
\hline 9 & $7.14(\mathrm{~d}, 7.3)$ & 122.6 & $7.28(\mathrm{~d}, 7.4)$ & 122.8 & $7.18(\mathrm{~d}, 7.4)$ & 121.2 \\
\hline 10 & $6.80(t, 7.3)$ & 120.2 & $7.01(t, 7.4)$ & 123.8 & $6.84(t, 7.4)$ & 120.6 \\
\hline 11 & $7.08(t, 7.3)$ & 128.4 & $7.11(\mathrm{t}, 7.4)$ & 127.4 & $7.09(t, 7.4)$ & 127.5 \\
\hline 12 & $6.71(\mathrm{~d}, 7.3)$ & 112.2 & $6.88(\mathrm{~d}, 7.4)$ & 114.2 & $6.75(\mathrm{~d}, 7.4)$ & 109.3 \\
\hline 13 & & 150.2 & & 145.8 & & 143.2 \\
\hline $14 \mathrm{a}$ & $1.55 \sim 1.51(\mathrm{~m})$ & 23.0 & $1.25 \sim 1.19(\mathrm{~m})$ & 16.0 & $1.59 \sim 1.51(\mathrm{~m})$ & 22.6 \\
\hline $14 \mathrm{~b}$ & $1.71 \sim 1.65(\mathrm{~m})$ & & $1.86 \sim 1.78(\mathrm{~m})$ & & $1.73 \sim 1.65(\mathrm{~m})$ & \\
\hline $15 \mathrm{a}$ & $1.15^{b}$ & 33.7 & $1.36^{b}$ & 36.4 & $1.26 \sim 1.22(\mathrm{~m})$ & 32.9 \\
\hline $15 b$ & $1.46 \sim 1.40(\mathrm{~m})$ & & $1.71 \sim 1.66(\mathrm{~m})$ & & $1.78 \sim 1.69(\mathrm{~m})$ & \\
\hline 16 & $2.90 \sim 2.84(\mathrm{~m})$ & 39.5 & $2.81^{b}$ & 41.8 & & 92.4 \\
\hline $17 \mathrm{a}$ & $1.63^{b}$ & 32.5 & $1.79 \sim 1.73(\mathrm{~m})$ & 33.6 & $2.22(\mathrm{~d}, 1.7)$ & 25.4 \\
\hline $17 \mathrm{~b}$ & $2.43^{b}$ & & $2.57 \sim 2.43(\mathrm{~m})$ & & $2.70 \sim 2.65(\mathrm{~m})$ & \\
\hline $18 \mathrm{a}$ & $1.48^{b}$ & 29.5 & $1.36^{b}$ & 32.4 & $0.55^{b}$ & 7.1 \\
\hline $18 \mathrm{~b}$ & $1.64^{b}$ & & $1.97 \sim 1.90(\mathrm{~m})$ & & & \\
\hline $19 \mathrm{a}$ & $1.13^{b}$ & 33.0 & $1.31 \sim 1.26(\mathrm{~m})$ & 34.0 & $0.57^{b}$ & 28.8 \\
\hline $19 \mathrm{~b}$ & $1.49^{b}$ & & $1.53 \sim 1.47(\mathrm{~m})$ & & $0.96 \sim 0.88(\mathrm{~m})$ & \\
\hline 20 & & 32.9 & & 32.6 & & 38.5 \\
\hline 21 & $2.54(\mathrm{~s})$ & 69.8 & $3.24(\mathrm{~s})$ & 70.5 & $2.58(\mathrm{~s})$ & 73.3 \\
\hline 22 & & 173.5 & & & & \\
\hline 1'a & $2.64 \sim 2.59(\mathrm{~m})$ & 41.7 & $2.38 \sim 2.31(\mathrm{~m})$ & 51.7 & $2.32(\mathrm{dd}, 16.4,5.7)$ & 46.7 \\
\hline $1^{\prime} b$ & $2.76(\mathrm{dd}, 18.2,5.9)$ & & $2.71(\mathrm{dd}, 6.0,6.5)$ & & $2.72 \sim 2.65(\mathrm{~m})$ & \\
\hline $2^{\prime}$ & & 207.1 & & 207.7 & & 207.3 \\
\hline $3^{\prime}$ & $2.51(\mathrm{~s})$ & 30.9 & $2.09(\mathrm{~s})$ & 30.7 & $2.10(\mathrm{~s})$ & 31.0 \\
\hline $\mathrm{COOCH}_{3}$ & & & & & $3.73(\mathrm{~s})$ & 51.0 \\
\hline $\mathrm{COOCH}_{3}$ & & & & & & 169.1 \\
\hline $\mathrm{COOH}$ & & & & 176.6 & & \\
\hline $\mathrm{NH}$ & $3.58(\mathrm{~s})$ & & & & & \\
\hline
\end{tabular}

${ }^{a}$ Compound 5 was measured in DMSO- $d_{6}, 6,7$ in $\mathrm{CDCl}_{3} .{ }^{b}$ Overlapped. 
表 3 生物碱对 LPS 诱导的 RAW 264.7 细胞中炎症因子产生的影响 ${ }^{a}$

Table 3 Effect of compounds on the production of cytokines in LPS-induced RAW 264.7 cells

\begin{tabular}{|c|c|c|c|c|}
\hline Compd. & Concentration $/\left(\mu \mathrm{g} \bullet \mathrm{mL}^{-1}\right)$ & $\mathrm{IL}-1 \beta /\left(\mathrm{ng} \cdot \mathrm{L}^{-1}\right)$ & $\mathrm{PGE} 2 /\left(\mathrm{ng} \cdot \mathrm{L}^{-1}\right)$ & $\mathrm{TNF}-\alpha /\left(\mathrm{ng} \cdot \mathrm{L}^{-1}\right)$ \\
\hline Control & - & $3.05 \pm 0.10$ & $9.18 \pm 0.05$ & $17.66 \pm 1.39$ \\
\hline LPS & - & $6.35 \pm 0.35^{\mathbf{\Lambda}}$ & $17.55 \pm 0.12^{\boldsymbol{\Lambda}}$ & $28.42 \pm 3.55^{\mathbf{\Lambda}}$ \\
\hline Deametasona & 10 & $3.65 \pm 0.16^{* *}$ & $15.32 \pm 0.73^{*}$ & $17.29 \pm 0.31^{*}$ \\
\hline \multirow{3}{*}{1} & 5 & $3.51 \pm 0.02 * *$ & $12.31 \pm 1.44^{*}$ & $18.25 \pm 2.95$ \\
\hline & 1 & $6.15 \pm 0.31$ & $13.05 \pm 1.50 *$ & $19.40 \pm 1.24$ \\
\hline & 0.4 & $6.90 \pm 0.31$ & $15.32 \pm 3.15$ & $22.29 \pm 0.71$ \\
\hline \multirow{3}{*}{2} & 5 & $5.44 \pm 1.23$ & $12.15 \pm 0.98 * *$ & $19.89 \pm 2.66$ \\
\hline & 1 & $5.71 \pm 0.90$ & $14.82 \pm 1.18$ & $20.71 \pm 1.59$ \\
\hline & 0.4 & $6.59 \pm 1.09$ & $16.88 \pm 0.72$ & $19.93 \pm 2.02$ \\
\hline \multirow{3}{*}{4} & 5 & $3.30 \pm 0.03^{* *}$ & $13.66 \pm 1.46$ & $18.11 \pm 2.59$ \\
\hline & 1 & $4.62 \pm 0.50^{*}$ & $13.95 \pm 1.46$ & $18.53 \pm 1.70$ \\
\hline & 0.4 & $5.33 \pm 0.44$ & $15.63 \pm 0.99$ & $19.39 \pm 2.70$ \\
\hline \multirow{3}{*}{6} & 5 & $4.72 \pm 0.42 *$ & $10.75 \pm 0.93 * *$ & $18.79 \pm 0.47$ \\
\hline & 1 & $5.53 \pm 0.21$ & $12.82 \pm 1.37^{*}$ & $19.60 \pm 1.91$ \\
\hline & 0.4 & $5.88 \pm 0.17$ & $14.09 \pm 1.55$ & $21.83 \pm 2.86$ \\
\hline \multirow{3}{*}{7} & 5 & $3.47 \pm 0.00^{* *}$ & $11.02 \pm 1.14^{* *}$ & $29.73 \pm 1.16$ \\
\hline & 1 & $4.40 \pm 0.46^{*}$ & $13.04 \pm 1.69$ & $21.55 \pm 1.69$ \\
\hline & 0.4 & $5.08 \pm 0.46$ & $13.36 \pm 1.67$ & $18.44 \pm 1.97$ \\
\hline 8 & 5 & $5.94 \pm 0.23$ & $17.41 \pm 0.08$ & $22.89 \pm 3.77$ \\
\hline 10 & 5 & $6.88 \pm 1.77$ & $15.50 \pm 0.77$ & $18.38 \pm 3.39$ \\
\hline \multirow{3}{*}{11} & 5 & $4.60 \pm 0.71$ & $14.57 \pm 1.31$ & $16.70 \pm 0.67 *$ \\
\hline & 1 & $5.32 \pm 0.50$ & $15.84 \pm 1.34$ & $19.19 \pm 1.20$ \\
\hline & 0.4 & $5.41 \pm 0.18$ & $15.84 \pm 1.27$ & $21.74 \pm 0.33$ \\
\hline \multirow{3}{*}{15} & 5 & $5.12 \pm 0.71$ & $15.13 \pm 2.52$ & $12.91 \pm 0.73^{*}$ \\
\hline & 1 & $5.89 \pm 0.47$ & $16.20 \pm 2.52$ & $15.39 \pm 0.17 *$ \\
\hline & 0.4 & $6.58 \pm 0.47$ & $17.13 \pm 2.14$ & $18.95 \pm 0.44$ \\
\hline \multirow{3}{*}{16} & 5 & $4.76 \pm 0.84$ & $10.21 \pm 0.05^{* *}$ & $27.07 \pm 2.36$ \\
\hline & 1 & $5.84 \pm 0.43$ & $13.14 \pm 1.18^{*}$ & $20.89 \pm 0.63$ \\
\hline & 0.4 & $6.53 \pm 0.43$ & $17.15 \pm 0.29$ & $22.45 \pm 2.95$ \\
\hline \multirow{3}{*}{18} & 5 & $4.37 \pm 0.63$ & $12.29 \pm 0.52 * *$ & $18.49 \pm 2.13$ \\
\hline & 1 & $5.65 \pm 0.42$ & $13.03 \pm 1.72$ & $19.97 \pm 1.27$ \\
\hline & 0.4 & $6.03 \pm 0.32$ & $13.63 \pm 1.43$ & $22.19 \pm 1.49$ \\
\hline \multirow{3}{*}{20} & 5 & $5.46 \pm 0.06$ & $14.24 \pm 1.00 *$ & $19.15 \pm 2.60 *$ \\
\hline & 1 & $5.51 \pm 0.36$ & $14.56 \pm 0.82 *$ & $19.64 \pm 1.58^{*}$ \\
\hline & 0.4 & $5.90 \pm 0.36$ & $12.97 \pm 1.72$ & $21.86 \pm 2.06$ \\
\hline
\end{tabular}

${ }^{a \mathbf{\Lambda}} p<0.01$ vs. control, ${ }^{*} p<0.05,{ }^{* *} p<0.01$ vs. LPS.

264.7 细胞的增殖.

\section{2 结论}

文献报道 aspidosperma 类型生物碱增加一个丙酮基 后, 比原型的生物碱具有更显著的抗肿瘤活性 ${ }^{[32,33]}$. 值 得注意的是, 本研究获得的 7 个在 C-5 位连接丙酮基的 生物碱与其原型成分相比, 显示出更强的抗炎活性, 如 生物碱 1, 4, 6 和 7 分别是生物碱 10, 11, 20 和 8 的 C-5 丙酮基衍生物，而前者比后者具有更强的抗炎活性. 尤 其是生物碱 1 和 7, 在 IL-1 $\beta$, PGE2 和 TNF- $\alpha$ 这三个指
标均比原型生物碱 10 和 8 显示出更优越的活性. 初步构 效提示丙酮基可能是其抗炎活性吲哚生物碱的增效基团. 本研究为进一步的结构修饰和药理学研究提供了线索.

\section{3 实验部分}

\section{1 仪器与试剂}

熔点由 WRX-4 显微熔点仪(上海易测仪器设备有限 公司)测定; 比旋光由 JASCO P-1020 全自动数字旋光仪 测定; 紫外光谱由 Shimadzu UV2401PC 紫外-可见光分 光光度仪测定; 红外光谱由 Bruker Tensor-27 傅里叶变 
换中红外光谱仪测定, $\mathrm{KBr}$ 压片; 电喷雾电离质谱由 Waters Xevo TQ-S 三重四级杆质谱仪测定; 高分辨电喷 雾电离质谱在 API QSTAR Pulsar i 串联四级杆飞行时间 质谱仪上测定; 一维和二维核磁共振在 Bruker AM-400, DRX-500 超导核磁共振波谱仪和 AVANCE III-600 超低 温探头超导核磁共振波谱仪上测定, TMS 作为内标; 柱 色谱硅胶(200 300 目, 青岛谱科分离材料有限公司生 成, 中国); Sephadex LH-20 葡聚糖凝胶(瑞典乌普萨拉 Amersham Pharmacia Biotech 公司, 瑞典)和 MCI 材料 (日本京东 Mitsubishi 化工生产, 日本); 反相中压填充材 料 RP-18, 粒径 20 45 $\mu \mathrm{m}$ (德国达姆施塔特 Merck 公司 生产, 德国); Agilent 1200 HPLC 液相色谱仪, 半制备色 谱柱 Agilent Zorbax SB-C18 (4.6 mm×250 mm, $3 \mathrm{~mL} /$ $\mathrm{min}$ ); 二极管阵列检测器; 薄层色谱 (TLC) 硅胶板 (GF254, 青岛谱科分离材料有限公司生成, 中国); 显色 剂为改良碘化铋钾 Dragendorff's 试剂, 体积分数为 $10 \%$ 的硫酸乙醇.

\section{2 植物材料}

云南荵木茎 2012 年采自云南省西双版纳. 经中国 科学院昆明植物研究所崔景云先生鉴定为荵木属植物 云南荵木(Kopsia officinalis), 标本(Cui20121113)保存于 中国科学院昆明植物研究所中国西部植物化学与植物 资源国家重点实验室.

\section{3 提取与分离}

云南荵木(K. officinalis) 茎干燥样品 $13 \mathrm{~kg}$, 粉碎后 在室温下用体积分数为 $90 \%$ 的甲醇冷浸提取 4 次, 每次 $48 \mathrm{~h}$, 合并提取液, $55{ }^{\circ} \mathrm{C}$ 减压蒸馏除去溶剂得到浸膏. 浸膏经体积分数为 $0.5 \%$ 盐酸水溶液溶解浸泡, 搅拌, 调 节 $\mathrm{pH}$ 至 $2 \sim 3$, 过滤. 滤液中加入乙酸乙酯, 用体积分 数为 $10 \%$ 氨水溶液将水层的 $\mathrm{pH}$ 调至 $9 \sim 10$, 萃取 3 次, 合并乙酸乙酯萃取液, 浓缩得总生物碱 $98 \mathrm{~g}$. 总生物碱 用 $150 \mathrm{~g}$ 硅胶拌样, $3 \mathrm{~kg}$ 硅胶进行柱层析(氯仿/甲醇, $V$ : $V=1: 0 \sim 0: 1$ )梯度洗脱, TLC 检测, 合并相同馏分得 到 6 个段 Fr.A Fr.F. Fr.A (1.5 g)经过硅胶柱色谱(石油 醚/丙酮, $V: V=4: 1)$ 洗脱得到生物碱 $8(56 \mathrm{mg})$ 和 $\mathbf{1 2}$ $(96 \mathrm{mg})$. Fr.B (2.7 g) 经 RP-18 中压柱色谱(甲醇/水, $V$ : $V=40 ： 60 \sim 70: 30$ )梯度洗脱, 后通过硅胶柱色谱(石 油醚/丙酮, $V: V=2: 1)$ 得到化合物 $\mathbf{1}(9 \mathrm{mg}), \mathbf{1 5}$ (35 $\mathrm{mg}$ )和 $10(10 \mathrm{mg})$. Fr.C (18 g) 通过中压柱色谱 RP-18(甲 醇/水, $V: V=20: 80 \sim 100: 0)$ 梯度洗脱, TLC 检测合 并相同馏分得到 3 小段 Fr.C1 Fr.C3. Fr.C1 通过半制备 $\mathrm{HPLC}$ (甲醇/水, $V: V=70: 30,2.5 \mathrm{~mL} / \mathrm{min}$ ) 等度洗脱得 到化合物 $17\left(14 \mathrm{mg}, t_{\mathrm{R}}=8 \mathrm{~min}\right), 3\left(24 \mathrm{mg}, t_{\mathrm{R}}=12.5 \mathrm{~min}\right)$ 和 16 (38 mg, $\left.t_{\mathrm{R}}=15 \mathrm{~min}\right)$. 化合物 $9(17 \mathrm{mg})$ 从 Fr.C2 中 结晶, 将其母液 $589 \mathrm{mg}$ 经 Sephadex LH-20, 甲醇洗脱得
到化合物 $22(35 \mathrm{mg})$ 和 $20(7 \mathrm{mg})$. Fr.C3 经硅胶柱色谱 (氯仿/丙酮, $V: V=10: 1 \sim 3: 1$ ) 洗脱, Sephadex LH-20 甲醇洗脱后得化合物 $\mathbf{5}(6 \mathrm{mg}), \mathbf{1 1}(98 \mathrm{mg}), \mathbf{1 3}(35 \mathrm{mg})$ 和 14 (498 mg). Fr.D (32.8 g) 经反相 RP-18 柱色谱(甲醇/水, $V: V=30: 70 \sim 80: 20)$ 得到 Fr.D1 和 Fr.D2. Fr.D1 经 Sephadex LH-20 柱色谱(氯仿/甲醇, $V: V=1: 1$ )得到化 合物 $18(78 \mathrm{mg})$ 和 $21(8 \mathrm{mg})$. Fr.D2 经硅胶柱色谱(氯仿/ 甲醇, $V: V=12: 1,3: 1)$ 得到化合物 $19(367 \mathrm{mg}), 2(26$ $\mathrm{mg})$ 和 $26(9 \mathrm{mg})$. Fr.E $(17 \mathrm{~g})$ 经硅胶柱色谱(氯仿/甲醇, $V: V=8: 1,2: 1$ ), Sephadex LH-20(甲醇)柱色谱和重 结晶得到化合物 $\mathbf{4}(22 \mathrm{mg}), \mathbf{6}(15 \mathrm{mg})$ 和 $27(12 \mathrm{mg})$. Fr.F $(15 \mathrm{~g}$ ) 经 RP-18(甲醇/水, $V: V=20: 80 \sim 100 ： 0$ ), Sephadex LH-20(甲醇)得到化合物 25 (109 mg), 进一步经 半制备 HPLC(乙腈/水, $V: V=45: 55 \sim 80: 20,2.5$ $\mathrm{mL} / \mathrm{min})$ 分离纯化得到化合物 $\mathbf{2 3}\left(56 \mathrm{mg}, t_{\mathrm{R}}=13 \mathrm{~min}\right), \mathbf{2 4}$ (34 mg, $\left.t_{\mathrm{R}}=14.5 \mathrm{~min}\right)$ 和 7 ( $\left.8 \mathrm{mg}, t_{\mathrm{R}}=16 \mathrm{~min}\right)$.

\section{4 抗炎活性测定}

巨噬细胞 RAW 264.7 在含体积分数为 $10 \%$ 新生牛 血清的 DMEM 培养基中培养, 于 $37{ }^{\circ} \mathrm{C}$ 体积分数为 $5 \%$ 的 $\mathrm{CO}_{2}, 100 \%$ 相对湿度下生长. 取对数生长期的 $\mathrm{RAW}$ 264.7 细胞, 经消化计数后, 以 $2 \times 10^{4} /$ 孔接种于 96 孔板 中, $24 \mathrm{~h}$ 后每个孔加不同浓度 $(5,1,0.4 \mu \mathrm{g} / \mathrm{mL})$ 的生物碱, $2 \mathrm{~h}$ 后加 LPS $\left(20 \mu \mathrm{mol} \cdot \mathrm{L}^{-1}\right)$, 用地塞米松 $(10 \mu \mathrm{g} / \mathrm{mL})$ 作为 阳性对照, 继续培养 $24 \mathrm{~h}$ 后. 收集培养基上清, 然后按 照试剂盒说明操作, 通过 ELISA 试剂盒(武汉华美生物 技术，武汉，中国)测试白细胞介素-1 $\beta$ (IL-1 $\beta$ )、前列腺 素 2 (PGE2)和肿瘤坏死因子 $-\alpha(\mathrm{TNF}-\alpha)$. 所有实验均在 没有细胞毒性的浓度内进行. 结果表示为平均值 \pm $\mathrm{SEM}, \mathrm{t}$ 检验确定统计学显著性, $p<0.01$ 或 $p<0.05$ 被认 为是有显著差异的.

\section{5 波谱数据}

Kopsiofficine A (1): 白色无定型粉末, m.p. 215 $217{ }^{\circ} \mathrm{C} ;[\alpha]_{\mathrm{D}}^{22.4}+23\left(c 0.15, \mathrm{CH}_{3} \mathrm{OH}\right) ; \mathrm{UV}\left(\mathrm{CH}_{3} \mathrm{OH}\right) \lambda_{\max }$

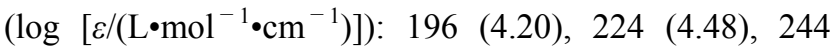
(4.09), 290 (3.28) nm; IR (KBr) v: 3438, 2928, 2865, 1756, $1683,1633,1469,1359,1249,1096,758,741 \mathrm{~cm}^{-1}$; HREIMS calcd for $\mathrm{C}_{26} \mathrm{H}_{29} \mathrm{~N}_{2} \mathrm{O}_{7}[\mathrm{M}+\mathrm{H}]^{+} 481.1969$, found 481.1965.

Kopsiofficine B (2): 无色油状物; $[\alpha]_{\mathrm{D}}^{22.4}+21(c$ $\left.0.12, \mathrm{CH}_{3} \mathrm{OH}\right) ; \mathrm{UV}\left(\mathrm{CH}_{3} \mathrm{OH}\right) \lambda_{\max }\left(\log \left[\varepsilon /\left(\mathrm{L}^{2} \mathrm{~mol}^{-1} \bullet\right.\right.\right.$ $\left.\mathrm{cm}^{-1}\right)$ ]): 220 (4.42); 240 (3.99); 274 (3.24) nm; IR (KBr) $v$ : 3425, 2929, 2863, 1751, 1708, 1654, 1473, 1375, 1244, 1061, 1052, $790 \mathrm{~cm}^{-1}$; HREIMS calcd for $\mathrm{C}_{24} \mathrm{H}_{27} \mathrm{~N}_{2} \mathrm{O}_{5}$ $[\mathrm{M}+\mathrm{H}]^{+}$423.1914, found 423.1914.

Kopsiofficine C (3): 无色油状物; $[\alpha]_{D}^{22.4}-25(c$ 


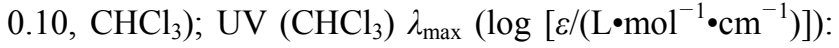
242 (3.83), 295 (3.51) nm; IR (KBr) v: 3439, 2929, 2861, 1738, 1611, 1479, 1459, 1089, $750 \mathrm{~cm}^{-1}$; HREIMS calcd for $\mathrm{C}_{23} \mathrm{H}_{27} \mathrm{~N}_{2} \mathrm{O}_{2}[\mathrm{M}+\mathrm{H}]^{+}$363.2067, found 363.2064.

Kopsiofficine D (4): 无色油状物; $[\alpha]_{\mathrm{D}}^{22.4}+17(c$ $\left.0.18, \mathrm{CH}_{3} \mathrm{OH}\right) ; \mathrm{UV}\left(\mathrm{CH}_{3} \mathrm{OH}\right) \lambda_{\max }\left(\log \left[\varepsilon /\left(\mathrm{L} \bullet \mathrm{mol}^{-1} \bullet\right.\right.\right.$ $\left.\left.\mathrm{cm}^{-1}\right)\right]$ ): 211 (4.45), 237 (3.97), 278 (3.30) nm; IR (KBr) $v$ : 3330, 2935, 2863, 1757, 1711, 1600, 1461, 1442, 1356, 1090, $756 \mathrm{~cm}^{-1}$; HREIMS calcd for $\mathrm{C}_{26} \mathrm{H}_{29} \mathrm{~N}_{2} \mathrm{O}_{6}[\mathrm{M}-\mathrm{H}]$ 465.2031, found 465.2042.

Kopsiofficine E (5): 无色油状物; $[\alpha]_{\mathrm{D}}^{22.4}+55(c$

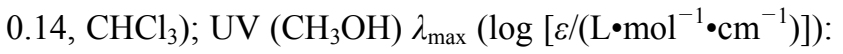
192 (3.26), 214 (3.35), 244 (3.80), 291 (3.43) nm; IR (KBr) v: 3446, 2926, 1716, 1638, 1458, 1384, 1257, 1195, $759,744 \mathrm{~cm}^{-1}$; HREIMS calcd for $\mathrm{C}_{23} \mathrm{H}_{26} \mathrm{~N}_{2} \mathrm{O}_{3} \mathrm{Na}[\mathrm{M}+$ $\mathrm{Na}]^{+}$401.1836, found 401.1836.

Kopsiofficine F (6): 白色无定型粉末; m.p. 165 $166{ }^{\circ} \mathrm{C} ;[\alpha]_{\mathrm{D}}^{22.4}-93\left(c 0.14, \mathrm{CH}_{3} \mathrm{OH}\right) ; \mathrm{UV}\left(\mathrm{CH}_{3} \mathrm{OH}\right) \lambda_{\max }$

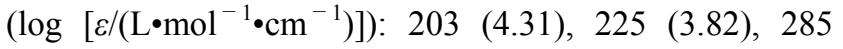
(3.31) nm; IR (KBr) v: 3440, 2926, 2864, 1713, 1631, 1608, 1461, 1383, 1049, $752 \mathrm{~cm}^{-1}$; HREIMS calcd for $\mathrm{C}_{23} \mathrm{H}_{29} \mathrm{~N}_{2} \mathrm{O}_{3}[\mathrm{M}+\mathrm{H}]^{+}$381.2173, found 381.2174.

Kopsiofficine $\mathrm{G}(7)$ : 无色油状物; $[\alpha]_{\mathrm{D}}^{22.4}+183(c$ 0.11, $\left.\mathrm{CH}_{3} \mathrm{OH}\right) ; \mathrm{UV}\left(\mathrm{CH}_{3} \mathrm{OH}\right) \lambda_{\max }\left(\log \left[\varepsilon /\left(\mathrm{L} \cdot \mathrm{mol}^{-1} \bullet\right.\right.\right.$ $\left.\mathrm{cm}^{-1}\right)$ ]): 205 (4.20), 299 (3.86), 326 (3.87) nm; IR (KBr) ) v: 3426, 2924, 1713, 1667, 1632, 1464, 1440, 1047, 878, 749, $706 \mathrm{~cm}^{-1}$; HREIMS calcd for $\mathrm{C}_{24} \mathrm{H}_{31} \mathrm{~N}_{2} \mathrm{O}_{3}[\mathrm{M}+\mathrm{H}]^{+}$ 395.2329, found 395.2326.

辅助材料(Supporting Information) 化合物 $1 \sim 7$ 的 1D/2D NMR, HRESIMS, UV, IR 和 ORD 谱图. 这些材料 可以免费从本刊网站(http://sioc-journal.cn/)上下载.

\section{References}

[1] Aggarwal, B.-B.; Shishir, S.; Sandur, S.-K.; Pandey, M.-K.; Gautam, S. Biochem. Pharmacol. 2006, 72, 1605.

[2] Xu, X. T.; Chen, J.; Lin, Z. Q.; Li, D, L.; Zhang, K.; Sheng, Z. J.; Wang, S. H.; Zhu, S.; Abdullah, M. A. Chin. J. Org. Chem. 2019, 39, 2958 (in Chinese).

(徐学涛, 陈洁, 林芷晴, 李冬利, 张焜, 盛到君, 王少华, 朱顺, Abdullah M. Asiri, 有机化学, 2019, 39, 2958.)

[3] Wellen, K.-E.; Hotamisligil, G.-S. J. Clin. Invest. 2005, 115, 1111.

[4] Gao, S. F.; Xu, Q. L.; Li, J. M.; Chu, Z. X.; He, G. W.; Lin, G. F.; Zhu, Z. W.; Cui, Y.; Mo, J. J.; Guo, J.; Zhao, Y. Chin. J. Org. Chem. 2018, 38, 478 (in Chinese).
(高粟繁，许勤龙，李家明，储昭兴，何广卫，林高峰，朱正伟， 崔勇, 莫佳佳, 郭敬, 赵炎, 有机化学, 2018, 38, 478.)

[5] Coussens, L.-M.; Werb, Z. Nature 2002, 420, 860.

[6] Harirforoosh, S.; Asghar, W.; Jamali, F. J. Pharm. Pharm. Sci. 2013, 16,821 .

[7] Gulati, A.; Bagga, A.; Gulati, S.; Mehta, K.-P.; Vijayakumar, M. Indian Pediatr. 2009, 46, 35.

[8] Zeng, J.; Zhang, D.-B.; Zhou, P.-P.; Zhang, Q.-L.; Zhao, L.; Chen, J.-J.; Gao, K. Org. Lett. 2017, 19, 3998.

[9] Yu, K.; Gao, B.-L.; Ding, H.-F. Acta Chim. Sinica 2016, 74, 410 (in Chinese). (余宽, 高北岭, 丁寒锋, 化学学报, 2016, 74, 410.)

[10] Zhang, D.; Qin, Y. Acta Chim. Sinica 2013, 71, 147 (in Chinese). (张丹, 秦勇, 化学学报, 2013, 71, 147.)

[11] Sistla, R.; Diwan, P.-V. Indian J. Pharm. Sci. 1999, 61, 275.

[12] Ye, X.-H.; Wu, H.; Sheng, L.-Y.; Liu, Y.-X.; Ye, F.; Wang, M.; Zhou, H.; Su, Y.; Zhang, X.-K. Nat. Commun. 2019, 10, 1.

[13] Huang, S.-P.; Wen, Y.-C.; Huang, S.-T.; Lin, C.-W.; Wang, T.-D.; Hsiao, F.-Y. Drug Safety 2019, 42, 67.

[14] Zeng, T.; Wu, X.-Y.; Yang, S.-X.; Lai, W.-C.; Shi, S.-D.; Zou, Q.; Liu, Y.; Li, L.-M. J. Nat. Prod. 2017, 80, 864.

[15] Wu, Y.-Q.; Suehiro, M.; Kitajima, M.; Matsuzaki, T.; Hashimoto, S.; Nagaoka, M.; Zhang, R.-P.; Takayama, H.; Takayama, H. J. Nat. Prod. 2009, 72, 204.

[16] Wenkert, E.; Cochran, D.-W.; Hagaman, E.-W.; Schell, F.-M.; Neuss, N.; Katner, A.-S.; Potier, P.; Kan, C.; Plat, M.; Koch, M.; Mehri, H.; Poisson, J.; Kunesch, N.; Holland, Y. J. Am. Chem. Soc. 1973, 95, 4990.

[17] Danieli, B.; Lesma, G.; Palmisano, G.; Riva, R.; Tollari, S. J. Org. Chem. 1984, 49, 547 .

[18] Kam T.-S.; Subramaniam, G.; Chen, W. Phytochemistry 1999, 51, 159.

[19] Lim, K.-H.; Low, Y.-Y.; Tan, G.-H.; Kam, T.-S. Helv. Chim. Acta 2010, $91,1559$.

[20] Feng. X.-Z.; Kan, C.; Potier, P.; Kan, S.-K.; Lounasmaa, M. Planta Med. 1983, 48, 280.

[21] Thomas, D.-W.; Achenbach, H.; Biemann, K. J. Am. Chem. Soc. 1966, 88, 3423.

[22] Kam, T.-S.; Sim, K.-M.; Koyano, T.; Komiyama, K. Phytochemistry 1999, 50, 75 .

[23] Feng, X.-Z; Kan, C.; Husson, H.-P.; Potier, P.; Kan, S.-K.; Lounasmaa, M. J. Nat. Prod. 1984, 47, 117.

[24] Subramaniam, G.; Hiraku, O.; Hayashi, M.; Koyano, T.; Komiyama, K.; Kam, T.-S. J. Nat. Prod. 2007, 70, 1783.

[25] Mariko, K.; Minako, A.; Noriyuki, K.; Sumphan, W.; Hiromitsu, T. Tetrahedron 2014, 70, 9099.

[26] Yap, W.-S.; Gan, C.-Y.; Sim, K.-S.; Lim, S.-H.; Low, Y.-Y.; Kam, T.-S. J. Nat. Prod. 2016, 79, 230.

[27] Yang, Y.; Zuo, W.-J.; Zhao, Y.-X.; Dong, W.-H.; Mei, W.-L.; Dai, H.-F. Planta Med. 2012, 78, 1881.

[28] Yang, C.-Q.; Ma, Y.-F.; Chen, Y.-G. Chem. Nat. Compd. 2017, 53, 595.

[29] Kam, T.-S.; Tan, P.-S. Phytochemistry 1990, 29, 2321

[30] Kam, T.-S.; Tan, P.-S. Phytochemistry 1995, 39, 469.

[31] Cai, X.-H.; Li, Y.; Liu, Y.-P.; Li, X.-N.; Bao, M.-F.; Luo, X.-D. Phytochemistry 2012, 83, 116.

[32] Liu, Y.-P.; Li, Y.; Cai, X.-H.; Li, X.-L.; Kong, L.-M.; Cheng, G.-G.; Luo, X.-D. J. Nat. Prod. 2012, 75, 220.

[33] Li, Y.; Zhao, Y.-L.; Zhou, X.; Ni, W.; Dai, Z.; Yang, D.; Hao, J.-J.; Luo, L.; Liu, Y.-P.; Luo, X.-D.; Zhao, X.-D. Toxins 2017, 9, 150. 